\title{
Treatment of stable slipped capital femoral epiphysis: systematic review and exploratory patient level analysis
}

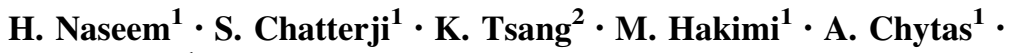 \\ S. Alshryda ${ }^{1}$ (i)
}

Received: 1 May 2017/ Accepted: 13 July 2017/Published online: 22 August 2017

(c) The Author(s) 2017. This article is an open access publication

\begin{abstract}
Background Several aspects of slipped capital femoral epiphysis (SCFE) treatment remain controversial. Loder's work has been instrumental in changing our understanding and approach to the management of the condition when he introduced the concept of "slip instability" and showed that avascular necrosis (AVN) developed in $47 \%$ of unstable slips but none of the stable slips. As the two types of SCFE behave differently in terms of presentation, progress and complications, we approached them as two different conditions to highlight these differences. This paper focuses on treatments of stable SCFE.

Materials and methods An extensive literature search was carried out from multiple databases. One thousand six hundred and twenty-three citations were screened. Three hundred and sixteen full publications were obtained for further scrutiny. Fifty-eight studies (2262 hips) were included in the review. These studies evaluated 6 interventions. AVN was chosen as a surrogate for bad outcome. Secondary outcomes were chondrolysis (CL), femoro-acetabular impingement (FAI), osteoarthritis (OA) and patients' reported outcomes. The latter were pooled when they met our predefined criteria.

Results The type of surgical intervention was an important risk factor. Pinning in situ (PIS) was associated with the lowest AVN rate $(1.4 \%)$. Moreover, the CL, FAI and OA rates were relatively low in patients who underwent PIS.
\end{abstract}

S. Alshryda

sattar26@doctors.org.uk

1 Royal Manchester Children's Hospital, Central Manchester Hospitals Foundation Trust, Oxford Road, Manchester, UK

2 University Hospitals of North Midlands, Royal Stoke Hospital, Stoke-on-Trent, UK
These were not translated into high patient satisfaction rates among these patients, with only $47 \%$ reporting an "excellent" outcome. In contrast, $87 \%$ of patients who underwent Ganz surgical dislocation reported an "excellent" outcome. The Ganz surgical dislocation was associated with an AVN rate of $3.3 \%$; double that observed in pinning in situ.

Conclusion Pinning in situ is the best treatment for mild and moderate stable slip. Ganz surgical dislocation gives higher patient satisfaction for severe stable slip but the risk of AVN is doubled compared with pinning in situ. Devices that allow continued growth may be better than standard screws.

Level of evidence Level III.

Keywords Slipped upper femoral epiphysis - Stable .

SUFE - SCFE · Unstable · Pinning in situ · Ganz surgical dislocation

\section{Introduction}

Slipped capital femoral epiphysis (SCFE) is an uncommon paediatric hip disorder occurring at an incidence of 1-10/ 100,000 . Despite it being uncommon, it is a condition which is important not to miss, as suboptimal management can lead to substantial disability. Various theories regarding the pathophysiology of this condition have been proposed and include increased shear forces acting on a weakened physis. Mechanical and hormonal factors have both been implicated [1].

Loder's work has been instrumental in changing our understanding and approach to the management of the condition. In a landmark paper [2], he categorised SCFEs into stable and unstable based on the patient's ability to 
ambulate (with or without crutches) or not. Almost half the patients with an unstable slip developed poor outcomes versus none in the stable group. This finding has been confirmed by several authors [3-6]. Although our knowledge of the condition has advanced over the last three decades, this has not translated into obviously better outcomes [7-20].

Various treatment options have been proposed, including bone peg epiphysiodesis, pinning in situ (PIS), closed reduction and pinning (CRIF), open reduction and physeal osteotomy (PO), open reduction and internal fixation (ORIF) and Ganz surgical dislocation (GSD). The types of fixation devices and their designs have been the subject of various researches. Ideal fixation devices should prevent further slippage, while allowing for continued growth with possible remodelling and prevention of future impingement [21-24]. The general consensus appears to be managing patients according to their slip grade. This was addressed in a review of the subject by Loder et al. in 2012, with mild and moderate slips tending to be treated with pinning in situ. Severe slips can be challenging to manage, as achieving a screw position centrally in the epiphysis with PIS may be technically difficult and subsequent remodelling may be insufficient.

With a low incidence, several treatment options and a potential lack of appropriate outcome measures, performing adequately powered randomised controlled trials (RCTs) is challenging. A nationwide study is currently underway, supported by the British Society of Children's Orthopaedic Surgery (the BOSS study), to help pave the way for future large-scale RCTs to inform decision making [25].

Given the substantial differences in the outcomes between stable and unstable slips we have chosen to study them separately, conducting two systematic reviews and patient level analysis. In a previous study we dealt with the outcomes of various interventions in treating unstable slips [26] and in this study we have critically appraised the published research to provide evidence on what may be the best current treatment for a stable slipped capital femoral epiphysis.

\section{Materials and methods}

This is a systematic review and patient level analysis of studies assessing the outcomes of interventions in stable slipped capital femoral epiphysis. As the concept of slip stability was introduced in 1993, studies before this date were not included. The work was conducted as part of a Cochrane Review and followed a prospective review protocol [27]. Reporting follows the PRISMA guidelines [28].
Avascular necrosis of the femoral head (AVN) was chosen as a surrogate for a poor outcome; this was our primary outcome measure $[1,29]$. The secondary outcome measures selected were osteoarthritis (OA), chondrolysis (CL), femoro-acetabular impingement (FAI) and surgical complications such as metalware problems, nerve palsy and infection. Several studies used patient reported outcome measures (PROMs) and these were also included in the analysis.

A hierarchical approach was used to include relevant studies. Randomised controlled trials (RCTs) or controlled clinical trials (CCTs) were included if adequately informative, otherwise inclusion would be firstly extended to controlled observational designs and secondly to other uncontrolled designs such as case series.

An extended literature search was performed of the following databases: Cochrane Bone, Joint and Muscle Trauma Review Group Specialised Register, the Cochrane Central Register of Controlled Trials (The Cochrane Library, current issue), MEDLINE (1993-2016), EMBASE (1993-2016), CINAHL (1993-2016), and Science Citation Index (ISI Web of Science 1993-2016). Table 1 summarises the search strategy for MEDLINE, which was modified for the other databases. The bibliographies of the retrieved literature were cross-referenced to identify other relevant studies.

Table 1 Search strategies

1. Epiphyses, Slipped/
2. (slipped adj3 upper adj3 femoral adj3 epiphysis).tw.
3. Femur Head/ab, pa, su [Abnormalities, Pathology, Surgery]
4. exp Femur Neck/ab, pa, su [Abnormalities, Pathology, Surgery]
5. SUFE.tw.
6. (slipped adj3 epiphyses).tw.
7. exp Slipped Capital Femoral Epiphyses/
8. SCFE.mp. or SCUFE.tw. [mp = title, abstract, original title,
name of substance word, subject heading word, protocol
supplementary concept, rare disease supplementary concept,
unique identifier]
9. or/1-8
10. randomized controlled trial.pt.
11. controlled clinical trial.pt.
12. randomized.ab.
13. placebo.ab.
14. drug therapy.fs.
15. randomly.ab.
16. trial.ab.
17. groups.ab.
18. or/10-17
19. exp animals/not humans.sh.
20. 18 not 19
21. 9 and 20


Table 2 Risk of bias assessment tool for cohort studies

\begin{tabular}{|c|c|c|c|}
\hline Domain & Items & $\begin{array}{l}\text { Maximum number of } \\
\text { stars }\end{array}$ & Notes \\
\hline \multirow[t]{4}{*}{ Selection } & 1. Representativeness of the exposed cohort & 1 & \multirow{4}{*}{$\begin{array}{l}\text { Maximum possible stars is } \\
4\end{array}$} \\
\hline & 2. Selection of the non exposed cohort & 1 & \\
\hline & 3. Ascertainment of exposure & 1 & \\
\hline & $\begin{array}{l}\text { 4. Demonstration that outcome of interest was not present at start of } \\
\text { study }\end{array}$ & 1 & \\
\hline Comparability & Comparability of cohorts on the basis of the design or analysis & 2 & $\begin{array}{l}\text { Maximum possible stars is } \\
2\end{array}$ \\
\hline \multirow[t]{3}{*}{ Outcome } & 1. Assessment of outcome & 1 & \multirow{3}{*}{$\begin{array}{l}\text { Maximum possible stars is } \\
3\end{array}$} \\
\hline & 2. Was follow-up long enough for outcomes to occur & 1 & \\
\hline & 3. Adequacy of follow-up of cohorts & 1 & \\
\hline
\end{tabular}

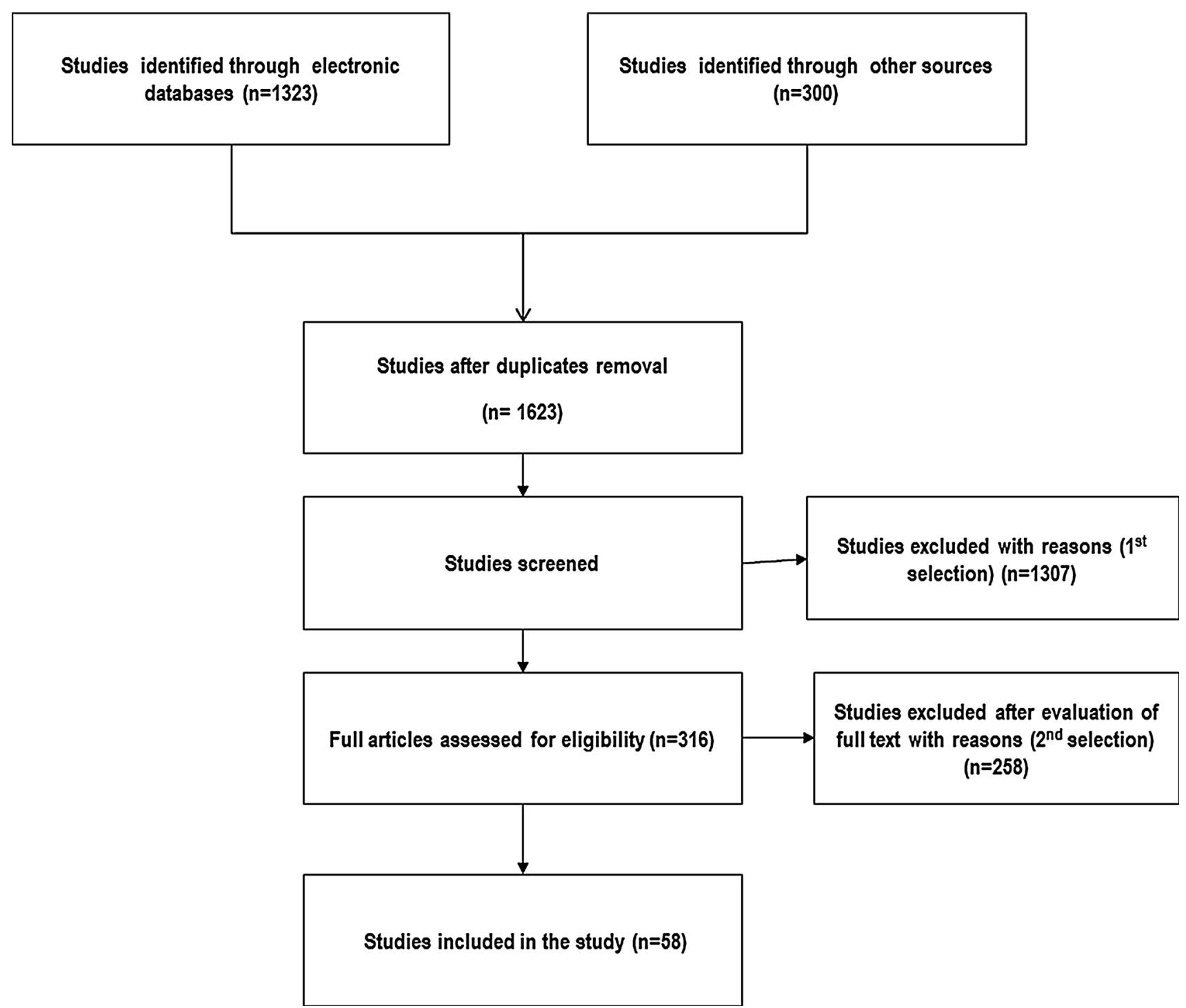

Fig. 1 Studies selection flow chart 
Table 3 Pooled summary of studies of stable slip treatments

\begin{tabular}{|c|c|c|c|c|c|c|}
\hline Intervention & Hips & $\operatorname{AVN}(\%)$ & CL $(\%)$ & FAI $(\%)$ & $\mathrm{OA}(\%)$ & Satisfactory patients result $\mathrm{t}^{\mathrm{a}}$ \\
\hline Hip spica & 101 & 9.5 & 20.5 & NR & 53 & NR \\
\hline Epiphysiodesis & 464 & 3 & 1.3 & NR & 23.3 & $\begin{array}{l}67(67 \%) \text { excellent } \\
6(6 \%) \text { good } \\
10(10 \%) \text { fair } \\
7(7 \%) \text { poor } \\
7(7 \%) \text { failure }\end{array}$ \\
\hline Pinning using single screw & 714 & 1.4 & 2.1 & 29.8 & 3.1 & $\begin{array}{l}116(47 \%) \text { excellent } \\
86(36 \%) \text { good } \\
19(8 \%) \text { fair } \\
10(4 \%) \text { poor } \\
11(5 \%) \text { failure }\end{array}$ \\
\hline Pinning using multiple pins & 273 & 2.2 & 4 & NR & 15 & $\begin{array}{l}76(67 \%) \text { excellent } \\
19(17 \%) \text { good } \\
0(0 \%) \text { fair } \\
16(14 \%) \text { poor } \\
3(3 \%) \text { failure }\end{array}$ \\
\hline Physeal osteotomy & 615 & 11.1 & 9.8 & 1.5 & 12.2 & $\begin{array}{l}131(28 \%) \text { excellent } \\
210(45 \%) \text { good } \\
46(10 \%) \text { fair } \\
72(16 \%) \text { poor } \\
3(6 \%) \text { failure }\end{array}$ \\
\hline Ganz surgical dislocation & 95 & 3.1 & 2.1 & 6 & 0 & $\begin{array}{l}52(87 \%) \text { excellent } \\
2(3 \%) \text { good } \\
0(0 \%) \text { fair } \\
5(8 \%) \text { poor } \\
1(2 \%) \text { failure }\end{array}$ \\
\hline
\end{tabular}

Percentage based on the number of patients in the studies that reported on the relevant outcomes and not the pooled total $A V N$ femoral head osteonecrosis, $C L$ chondrolysis, FAI femora-acetabular impingement, $O A$ osteoarthritis

a Satisfactory patients result based on closely related ratings such as Heyman and Herndon classification, Harris hip score or Iowa hip scores, $N R$ not reported

The above search strategy was independently applied by two reviewers (HN and SC) to identify studies. The article titles and abstracts were then independently reviewed. Full articles were obtained if the study appeared to be eligible or where this was uncertain. If necessary, authors were contacted for further information and clarification. Our senior authors (KT, AC and SA) were consulted if there was still a disagreement regarding inclusion. If no consensus was reached the study was excluded. Several studies were excluded because they were published more than once with more patients: it was agreed to include the most informative one regardless of the number of patients that were included.

A piloted form was used to extract data independently by two authors (KT and $\mathrm{MH}$ ). The names of included papers' authors or institutions were not masked. The data accuracy was jointly double-checked by these two authors and any discrepancies resolved through discussion. The two authors independently assessed the risk of bias in the included studies. The methodological quality of non-randomised studies (NRSs) was assessed using the NewcastleOttawa Scale (see Table 2).

The continuous data was reported for each trial arm as mean, standard deviation (SD) and group size. We planned to use the mean difference (MD) with corresponding $95 \%$ confidence interval (CI) to summarise trial findings and report the treatment effect if the outcomes were measured the same way between trials. The standardised mean difference (SMD) would be used to compare trials that measured the same outcome (construct), but used different scales. The dichotomous data was expressed as proportions or risks, reporting the treatment effect as a risk ratio (RR) with $95 \%$ CI. $P<0.05$ was selected as the level for statistical significance.

Various types of patient satisfaction scores were utilized in the included studies. These are summarised in Table 4. 
They were categorised into an ordinal scale (excellent, good, fair, poor and failure) by most studies. Authors were not consistent in how they used and reported them. Carlioz et al. [30] used a scale omitting "excellent". A few authors omitted "failure" in their scales [31-33]. We pooled data as reported in the included studies without assumption or improvisation.

\section{Results}

\section{Description of studies}

A total of 1623 potentially relevant citations were identified, of which 1307 were subsequently excluded for reasons such as duplications, reviews and commentaries. The full publications for the remaining 316 citations were obtained and of these 271 studies were further excluded: the main reasons included uncertainty of slip stability, the inability to link patients to outcomes within the study or that the focus of the study was not on outcomes. Forty-five studies were hence used in the review. This process is illustrated in Fig. 1. No RCTs were identified and all were retrospective case series or controlled studies. These scored between 2 and 4 stars (out of 7) on the risk of bias measure.

The treatment options identified were hip spica, bone graft epiphysiodesis, pinning in situ (PIS) pinning using multiple pins, physeal osteotomy (PO) and Ganz surgical dislocation (GSD). Several studies used more than one treatment option. Pinning in situ was the commonest treatment option seen. Patients were excluded from the analysis if there was uncertainty about factors such as the severity of the slip and the occurrence and/or type of reduction. Studies of base of neck osteotomy and intertrochanteric osteotomy were excluded from the review because they included a significant number of healed SCFEs.

In total, 2262 hips drawn from 58 studies were included in the review. Several studies reported on more than one treatment option. Table 3 summarises the included studies and categorises them according to the treatment methods. Three studies reported on 101 hips that were treated with hip spica [34-36] (Table 4). Six studies reported on 464 hips that were treated with bone peg epiphysiodesis [37-42] (Table 5). Nineteen studies (714 hips) reported on pinning in situ using a single screw $[3,23,30-32,43-55$, 65] (Table 6). Six studies (273 hips) reported on fixation in situ using multiple smooth pins [35, 42, 44, 45, 47, 56] (Table 7). Seventeen studies (615 hips) reported on primary corrective subcapital femoral osteotomy [3, 30, 33, 35, 41, 56-66, 70] (Table 8). Seven studies (95 hips) reported on safe surgical dislocation using the Ganz technique [43, 51, 52, 67-70] (Table 9). Although nine studies reported on screws that allow continued growth, only three met our inclusion criteria $[23,54,55]$. These were further analysed for their effect on growth of the femoral neck.

Table 4 Studies of hip spica treatment in stable slips

\begin{tabular}{|c|c|c|c|c|c|c|c|c|c|}
\hline Study & Patients & Hips & AVN & $\mathrm{CL}$ & FAI & $\mathrm{OA}$ & Patient satisfaction $^{a}$ & $\begin{array}{l}\text { Other } \\
\text { complications }\end{array}$ & Notes \\
\hline $\begin{array}{l}\text { Betz } \\
\text { [34] }\end{array}$ & 32 & 37 & 0 & 5 & NR & NR & NR & & $\begin{array}{l}0 \text { acute, } 8 \text { acute on chronic and } 29 \\
\text { chronic } \\
25 \text { mild, } 7 \text { moderate and } 5 \text { severe } \\
\text { All stable slips }\end{array}$ \\
\hline $\begin{array}{c}\text { Carney } \\
{[35]}\end{array}$ & NR & 47 & 8 & 6 & NR & NR & $\begin{array}{l}\text { Mean IHS } 65 \text { when SCFEs were } \\
\text { reduced and } 83 \text { when SCFEs } \\
\text { were not reduced }\end{array}$ & & $\begin{array}{l}\text { Spica with closed reduction (16 hips) } \\
\text { resulted in a mean IHS of } 65 \text { points, } \\
6 \text { AVN and } 2 \text { CL } \\
\text { Spica cast without reduction [26] } \\
\text { resulted in a mean IHS of } 83 \text { points, } \\
2 \text { AVN and } 4 \text { CL }\end{array}$ \\
\hline $\begin{array}{r}\text { Meier } \\
{[36]}\end{array}$ & 13 & 17 & NR & 10 & NR & 9 & NR & $\begin{array}{l}3 \text { pressure } \\
\text { sores } \\
3 \text { further } \\
\text { slipping }\end{array}$ & \\
\hline
\end{tabular}

$\begin{array}{llllllll}\text { Total } & \text { NR } & 101 & 8 & 21 & \text { NR } & 9 & \text { NR }\end{array}$

AVN rate $9.5 \%(8 / 84)$. CL rate $20.2 \%$ (21/101), FAI (NR), OA 53\% (9/17)

$A V N$ femoral head osteonecrosis, $C L$ chondrolysis, $N R$ not reported or suboptimum reporting to provide useful information, $I H S$ Iowa hip-rating system; excellent 90-100 points; good 80-89 points; fair 70-79 points; and poor $<70$ points, FAI femora-acetabular impingement, $O A$ osteoarthritis, SCFEs slipped capital femoral epiphyses

a Satisfactory patients result based on closely related ratings such as Heyman and Herndon classification, Harris hip score or Iowa hip scores 
Table 5 Studies of using bone peg epiphysiodesis in stable slips

\begin{tabular}{|c|c|c|c|c|c|c|c|c|c|}
\hline Study & Patients & Hips & AVN & CL & FAI & $\mathrm{OA}$ & $\begin{array}{l}\text { Patient } \\
\text { satisfaction }^{\mathrm{a}}\end{array}$ & Other complications & Notes \\
\hline $\begin{array}{l}\text { Adamczyk } \\
{[38]}\end{array}$ & 225 & 278 & 4 & 0 & NR & NR & NR & $\begin{array}{l}17 \text { further slipping } \\
4 \text { deep infection } \\
12 \text { re-operation }\end{array}$ & $\begin{array}{l}45 \text { acute, } 0 \text { acute on chronic and } 278 \text { chronic } \\
\text { Outcomes of acute slips were excluded } \\
\text { The average length of surgery was } 90 \text { min and } \\
\text { blood loss was } 200 \mathrm{ml} \text {. No blood transfusion } \\
\text { Iliac crest bone autograft was used }\end{array}$ \\
\hline $\begin{array}{c}\text { Murray } \\
{[39]}\end{array}$ & 31 & 42 & 4 & 0 & NR & NR & NR & $\begin{array}{l}2 \text { re-operation } \\
2 \text { wound healing } \\
\text { problems }\end{array}$ & $\begin{array}{l}3 \text { unstable slips were excluded } \\
\text { Average operative time was } 87 \text { min and blood } \\
\text { loss was } 148 \mathrm{ml} \text {. No blood transfusion } \\
\text { Fibular allograft with demineralised bone } \\
\text { matrix was used }\end{array}$ \\
\hline Rao [37] & 43 & 46 & 3 & 2 & NR & NR & NR & $\begin{array}{l}3 \text { infections } \\
7 \text { cases of transient } \\
\text { anterolateral thigh } \\
\text { hypesthesia } \\
44 \text { heterotopic } \\
\text { ossification }\end{array}$ & $\begin{array}{l}18 \text { unstable (excluded) and } 46 \text { stable slips } \\
\text { The average operating time and blood loss per } \\
\text { hip were } 122 \pm 34 \mathrm{~min} \text { and } 426 \pm 238 \mathrm{ml} \text {, } \\
\text { respectively }\end{array}$ \\
\hline $\begin{array}{l}\text { Schmidt } \\
{[40]}\end{array}$ & 33 & 40 & 1 & 1 & NR & NR & $\begin{array}{l}35 \text { excellent } \\
1 \text { good } \\
2 \text { fair } \\
2 \text { poor }\end{array}$ & $\begin{array}{l}1 \text { femoral neck } \\
\text { fracture } \\
1 \text { sub-trochanteric hip } \\
\text { fracture } \\
2 \text { coxa vara }\end{array}$ & $\begin{array}{l}31 \text { mild, } 9 \text { moderate, } 0 \text { severe } \\
6 \text { unstable and } 34 \text { were stable } \\
\text { The average time } 1 \mathrm{~h} 57 \mathrm{~min} \text { and blood loss } \\
\text { averaged } 360 \mathrm{ml} \\
\text { Allograft used }\end{array}$ \\
\hline $\begin{array}{l}\text { Szypryt } \\
{[41]}\end{array}$ & 25 & 30 & 2 & 3 & NR & 7 & $\begin{array}{l}12 \text { excellent } \\
5 \text { good } \\
8 \text { fair } \\
4 \text { poor }\end{array}$ & 3 wound infection & $\begin{array}{l}1 \text { acute, } 13 \text { acute-on-chronic, } 16 \text { chronic } \\
0 \text { mild, } 12 \text { moderate, } 18 \text { severe }\end{array}$ \\
\hline $\begin{array}{c}\text { Zahrawi } \\
\text { [42] }\end{array}$ & & 28 & 0 & 0 & NR & NR & $\begin{array}{l}20 \text { excellent } \\
0 \text { good } \\
0 \text { fair } \\
1 \text { poor } \\
7 \text { failure }\end{array}$ & $\begin{array}{l}4 \text { wound infection } \\
2 \text { graft failure } \\
1 \text { further slipping } \\
6 \text { needed further } \\
\text { surgery }\end{array}$ & $\begin{array}{l}\text { Severity (mean slip angle } 30 \text { ) } \\
\text { LOS } 21 \\
\text { Duration of surgery } 150 \mathrm{~min} \\
\text { Blood loss } 500 \mathrm{ml}\end{array}$ \\
\hline Total & NR & 464 & 14 & 6 & NR & NR & $\begin{array}{l}67(68 \%) \\
\text { excellent } \\
6(6 \%) \\
\text { good } \\
10(10 \%) \\
\text { fair } \\
7(7 \%) \text { poor } \\
7(7 \%) \\
\text { failure }\end{array}$ & $\begin{array}{l}16 \text { wound infection } \\
20 \text { further slipping } \\
22 \text { re-operation }\end{array}$ & $\begin{array}{l}\text { AVN rate } 3 \%(14 / 464) \text {. CL rate } 1.3 \%(6 / 464) \text {. } \\
\text { FAI (NR), OA } 23.3 \%(7 / 30)\end{array}$ \\
\hline
\end{tabular}

$A V N$ femoral head osteonecrosis, $C L$ chondrolysis, $C R I F$ closed reduction and internal fixation, $N R$ not reported or suboptimum reporting to provide useful information, LOS length of stay, FAI femora-acetabular impingement, $O A$ osteoarthritis

a Satisfactory patients result based on closely related ratings such as Heyman and Herndon classification, Harris hip score or Iowa hip scores

\section{Outcomes}

\section{Femoral head osteonecrosis}

Data on the development of AVN was provided for 2162 hips. Of these, 109 hips (5\%) developed AVN. The lowest rate of AVN was observed in the pinning in situ group using a single screw $(1.5 \%)$ and the highest rate was observed in patients who underwent physeal osteotomy $(11.1 \%)$. The different rates between interventions were statistically different $\left[\chi^{2}\right.$ test $(d f=5)$ : $P<0.001]$. 
Table 6 Studies of pinning in situ using screws

\begin{tabular}{|c|c|c|c|c|c|c|c|c|c|}
\hline Study & Patients & Hips & AVN & $\mathrm{CL}$ & FAI & $\mathrm{OA}$ & $\begin{array}{l}\text { Patient } \\
\text { satisfaction }^{\mathrm{a}}\end{array}$ & $\begin{array}{l}\text { Other } \\
\text { complications }\end{array}$ & Notes \\
\hline $\begin{array}{l}\text { Abu } \\
\text { Amara } \\
{[65]}\end{array}$ & NR & 37 & 1 & 1 & 30 & NR & NR & & $\begin{array}{l}\text { See physeal osteotomy } \\
\text { FAI diagnosis is based on radiological signs. } \\
\text { WOMAC (10) } \\
\text { HHS (86) }\end{array}$ \\
\hline $\begin{array}{l}\text { Alshryda } \\
\text { [3] }\end{array}$ & 36 & 36 & 1 & 1 & NR & NR & NR & 1 loss of fixation & $\begin{array}{l}\text { Unstable and uncertain hips were excluded. See } \\
\text { physeal osteotomy below }\end{array}$ \\
\hline $\begin{array}{l}\text { Aronson } \\
\text { [44] }\end{array}$ & 34 & 43 & 1 & 0 & NR & 1 & $\begin{array}{l}27 \text { excellent } \\
12 \text { good } \\
2 \text { fair } \\
2 \text { poor }\end{array}$ & $\begin{array}{l}2 \text { loss of fixation } \\
1 \text { sub-trochanteric } \\
\text { fracture } \\
2 \text { failed screw } \\
\text { removals }\end{array}$ & $\begin{array}{l}6 \text { acute and } 37 \text { chronic } \\
27 \text { mild, } 8 \text { moderate and } 8 \text { severe } \\
\text { See pinning using multiple fine wires }\end{array}$ \\
\hline $\begin{array}{c}\text { Blanco } \\
{[45]}\end{array}$ & 80 & 43 & 0 & 0 & NR & NR & NR & $\begin{array}{l}2 \text { metalware } \\
\text { problems } \\
1 \text { reoperation }\end{array}$ & $\begin{array}{l}1 \text { acute, } 6 \text { acute on chronic, } 36 \text { chronic } \\
23 \text { mild, } 12 \text { moderate, } 8 \text { severe } \\
1 \text { CRIF } \\
\text { See pinning using multiple fine wires }\end{array}$ \\
\hline $\begin{array}{c}\text { Carlioz } \\
{[30]}\end{array}$ & 34 & 38 & 0 & 2 & NR & NR & $\begin{array}{l}31 \text { good } \\
10 \text { fair } \\
2 \text { bad } \\
3 \text { failure }\end{array}$ & $\begin{array}{l}1 \text { sub-trochanteric } \\
\text { fracture }\end{array}$ & $\begin{array}{l}6 \text { patients underwent reduction ( } 1 \text { AVN excluded) } \\
\text { Authors did not use "excellent" in outcomes }\end{array}$ \\
\hline $\begin{array}{l}\text { Dan } \\
\text { Cosma } \\
{[43]}\end{array}$ & 6 & 6 & 0 & 0 & 2 & NR & NR & $\begin{array}{l}3 \text { metalware } \\
\text { removal } \\
\text { One re-slip after } \\
\text { metalware } \\
\text { removal } \\
\text { requiring fixation }\end{array}$ & $\begin{array}{l}4 \text { patients with unstable slips were excluded } \\
8 \text { had excellent and good results (stable and } \\
\text { unstable slips) } \\
\text { HHC }\end{array}$ \\
\hline $\begin{array}{l}\text { de Poorter } \\
\text { [46] }\end{array}$ & 61 & 78 & 2 & NR & 4 & 3 & NR & 5 THR & $\begin{array}{l}\text { Long-term follow-up (18 years) } \\
\text { HOOS (71) } \\
\text { EQ5D score }(0.83) \\
\text { EQ5D (VAS) } 79 \%\end{array}$ \\
\hline $\begin{array}{c}\text { Escott } \\
{[31]}\end{array}$ & 64 & 91 & NR & NR & NR & NR & $\begin{array}{l}15 \text { excellent } \\
39 \text { good } \\
8 \text { fair } \\
2 \text { poor }\end{array}$ & & $\begin{array}{l}\text { Long-term follow-up ( } 20 \text { years) } \\
\text { HHS }(84.9) \\
\text { SF12 (50) } \\
\text { UCLA }(7.3)\end{array}$ \\
\hline $\begin{array}{l}\text { Gonzalez- } \\
\quad \text { Moran } \\
{[47]}\end{array}$ & 25 & 31 & 1 & 0 & NR & NR & NR & $\begin{array}{l}1 \text { wound infection } \\
3 \text { metalware } \\
\text { problems }\end{array}$ & $\begin{array}{l}\text { All received two weeks of skin longitudinal } \\
\text { traction then pinning in situ without } \\
\text { manipulation } \\
22 \text { cases had a single screw and } 9 \text { had } 2 \text { screws } \\
11 \text { acute, } 6 \text { acute on chronic and } 14 \text { chronic } \\
1 \text { preslip, } 17 \text { mild, } 11 \text { moderate and } 2 \text { severe }\end{array}$ \\
\hline $\begin{array}{l}\text { Guzzanti } \\
\text { [23] }\end{array}$ & 10 & 6 & 0 & 0 & NR & NR & 6 excellent & & $\begin{array}{l}4 \text { unstable slips were excluded from the analysis. } 3 \\
\text { mild, } 3 \text { moderate and } 0 \text { severe } \\
\text { Authors used the modified AO cannulated screw } \\
\text { (HIT-MEDICA, Rimini, Italy) had a distal } \\
\text { segment with the original six threads reduced to } \\
\text { three which were } 9 \mathrm{~mm} \text { long and } 6.4 \mathrm{~mm} \text { in } \\
\text { diameter }\end{array}$ \\
\hline $\begin{array}{l}\text { Holmdahl } \\
\text { [55] }\end{array}$ & 13 & 13 & 0 & NR & NR & NR & NR & & $\begin{array}{l}3 \text { unstable slips were excluded. Authors used } \\
\text { Hansson pin }\end{array}$ \\
\hline
\end{tabular}


Table 6 continued

\begin{tabular}{|c|c|c|c|c|c|c|c|c|c|}
\hline Study & Patients & Hips & AVN & $\mathrm{CL}$ & FAI & $\mathrm{OA}$ & $\begin{array}{l}\text { Patient } \\
\text { satisfaction }^{\mathrm{a}}\end{array}$ & $\begin{array}{l}\text { Other } \\
\text { complications }\end{array}$ & Notes \\
\hline $\begin{array}{c}\text { Herman } \\
\text { [48] }\end{array}$ & 11 & 11 & 0 & 1 & NR & NR & $\begin{array}{l}11 \text { excellent } \\
0 \text { good } \\
0 \text { fair } \\
0 \text { poor }\end{array}$ & No further slipping & $\begin{array}{l}4 \text { acute, } 11 \text { acute on chronic, and } 6 \text { chronic } \\
\text { HHS ( } 95 \text { points) }\end{array}$ \\
\hline $\begin{array}{l}\text { Kenny } \\
{[32]}\end{array}$ & 40 & 53 & 0 & 1 & NR & 1 & $\begin{array}{l}\text { (31) } 58 \% \\
\text { excellent } \\
\text { (19) } 36 \% \\
\text { good } \\
\text { (2) } 4 \% \text { fair } \\
\text { (1) } 2 \% \text { poor }\end{array}$ & $\begin{array}{l}1 \text { sub-trochanteric } \\
\text { fracture } \\
\text { No further slipping }\end{array}$ & $\begin{array}{l}3 \text { acute, } 8 \text { acute on chronic and } 35 \text { chronic } \\
80 \% \text { mild, } 12 \% \text { moderate and } 2 \% \text { severe } \\
\text { HHC }\end{array}$ \\
\hline $\begin{array}{r}\text { Koval } \\
{[49]}\end{array}$ & 49 & 67 & 2 & 7 & NR & 2 & NR & $\begin{array}{l}1 \text { growing off } \\
\text { fixation } \\
1 \text { stress fracture of } \\
\text { the femoral neck }\end{array}$ & $\begin{array}{l}12 \text { acute, } 1 \text { acute on chronic, } 67 \text { chronic } \\
55 \text { mild, } 19 \text { moderate and } 6 \text { severe } \\
3 \text { CRIF ( } 1 \mathrm{AVN})\end{array}$ \\
\hline $\operatorname{Lim}[50]$ & 13 & 13 & 1 & 0 & & & $\begin{array}{l}8 \text { excellent } \\
2 \text { good } \\
2 \text { fair } \\
0 \text { poor } \\
1 \text { failure }\end{array}$ & & $\begin{array}{l}\text { All underwent preoperative traction } \\
\text { All acute or acute on chronic } \\
\left.\text { Severity: mean } 30^{\circ} \text { (range } 0^{\circ}-60^{\circ}\right) \\
\text { Aadalen criteria }\end{array}$ \\
\hline $\begin{array}{c}\text { Novais } \\
{[51]}\end{array}$ & 15 & 15 & 1 & & & & $\begin{array}{l}3 \text { excellent } \\
1 \text { good } \\
1 \text { fair } \\
3 \text { poor } \\
7 \text { failure }\end{array}$ & $\begin{array}{l}2 \text { metalware } \\
\text { problems } \\
1 \text { further slipping }\end{array}$ & $\begin{array}{l}\text { All patients had stable severe slip revealed better } \\
\text { deformity correction with the modified Dunn } \\
\text { procedure compared with in situ pinning } \\
\text { HHC }\end{array}$ \\
\hline $\begin{array}{c}\text { Souder } \\
{[52]}\end{array}$ & NR & 64 & 0 & 0 & NR & NR & NR & $\begin{array}{l}3 \text { metalware } \\
\text { problems } \\
1 \text { infection } \\
1 \text { further slipping }\end{array}$ & $\begin{array}{l}\text { Ganz surgical dislocation } \\
7 \text { unstable cause } 3 \text { AVN excluded }\end{array}$ \\
\hline Ward [53] & 42 & 53 & 0 & 0 & NR & NR & NR & $\begin{array}{l}1 \mathrm{HO} \\
2 \text { metalware } \\
\text { problems }\end{array}$ & $\begin{array}{l}2 \text { acute, } 3 \text { acute on chronic and } 48 \text { chronic } \\
19 \text { mild, } 25 \text { moderate and } 9 \text { severe } \\
5 \text { CRIF }\end{array}$ \\
\hline $\begin{array}{c}\text { Wensaas } \\
\text { [54] }\end{array}$ & 14 & 16 & 0 & 0 & NR & NR & NR & $\begin{array}{l}\text { No metalware } \\
\text { problem reported }\end{array}$ & $\begin{array}{l}2 \text { unstable slips were excluded } \\
\text { Authors used a modified Olmeda screw (De Puy) }\end{array}$ \\
\hline Total $(\%)$ & NA & 714 & 10 & 12 & 36 & & $\begin{array}{l}119 \\
\text { excellent } \\
86 \text { good } \\
19 \text { fair } \\
10 \text { poor } \\
11 \text { failure }\end{array}$ & & $\begin{array}{l}\text { AVN rate } 1.4 \%(10 / 714) \\
\text { CL rate } 2.0 \%(12 / 590) \\
\text { FAI } 28.9 \%(36 / 121) \\
\text { OA } 3.1 \%(6 / 195)\end{array}$ \\
\hline
\end{tabular}

$A V N$ femoral head osteonecrosis, $C L$ chondrolysis, $C R I F$ closed reduction and internal fixation, $N R$ not reported or suboptimum reporting to provide useful information, HHS Harris hip score or modified Harris hip score; excellent 90-100 points; good 80-89 points; fair 70-79 points; and poor $<70$ points, HHC Heyman and Herndon classification, WOMAC Western Ontario and McMaster Universities Osteoarthritis Index, FAI femora-acetabular impingement, $O A$ osteoarthritis

a Satisfactory patients result based on closely related ratings such as Heyman and Herndon classification, Harris hip score or Iowa hip scores

\section{Chondrolysis}

An overall 108 out of 2071 hips (5.2\%) developed chondrolysis (CL). The lowest rate of CL (1.3\%) was observed in patients who underwent bone peg epiphysiodesis and the highest $(20.5 \%)$ in patients who were treated with hip spica. The different rates between interventions were statistically different [ $\chi^{2}$ test $\left.(d f=5): P<0.001\right]$. Table 4 
Table 7 Studies of using multiple fine pins in stable slips

\begin{tabular}{|c|c|c|c|c|c|c|c|c|c|}
\hline Study & Patients & Hips & AVN & $\mathrm{CL}$ & FAI & $\mathrm{OA}$ & $\begin{array}{l}\text { Patient } \\
\text { satisfaction }^{\mathrm{a}}\end{array}$ & Other complications & Notes \\
\hline $\begin{array}{l}\text { Aronson } \\
\text { [44] }\end{array}$ & 39 & 54 & 2 & 3 & NR & 18 & $\begin{array}{l}27 \text { excellent } \\
13 \text { good } \\
0 \text { fair } \\
13 \text { poor } \\
1 \text { failure }\end{array}$ & $\begin{array}{l}13 \text { patients had pin } \\
\text { protruding through } \\
\text { the back of the } \\
\text { neck }\end{array}$ & $\begin{array}{l}4 \text { acute and } 50 \text { chronic } \\
34 \text { mild, } 14 \text { moderate and } 6 \text { severe } \\
\text { HHC }\end{array}$ \\
\hline $\begin{array}{c}\text { Blanco } \\
{[45]}\end{array}$ & NR & 25 & 1 & 0 & NR & NR & NR & $\begin{array}{l}8 \text { metalware } \\
\text { problems } \\
1 \text { growing off } \\
4 \text { reoperation }\end{array}$ & $\begin{array}{l}1 \text { preslip, } 4 \text { acute, } 6 \text { acute on chronic, } 12 \text { chronic } \\
11 \text { mild, } 9 \text { moderate, } 4 \text { severe } \\
7 \text { CRIF }\end{array}$ \\
\hline $\begin{array}{c}\text { Carney } \\
{[47]}\end{array}$ & NR & 37 & 3 & 1 & NR & NR & & & $\begin{array}{l}3 \text { acute and } 34 \text { chronic } \\
\text { Reduction and pinning resulted in a mean ISH of } \\
75 \text { points, } 2 \text { AVN, } 1 \text { CL. Pinning in situ resulted } \\
\text { in a mean IHS of } 85 \text { points, } 1 \text { AVN, } 0 \text { CL } \\
\text { IHS for chronic slips } 86 \text { and } 93 \text { for acute slips }\end{array}$ \\
\hline $\begin{array}{l}\text { Dreghorn } \\
{[56]}\end{array}$ & NR & 66 & 0 & 2 & NR & 0 & NR & $\begin{array}{l}1 \text { growing off } \\
\text { fixation }\end{array}$ & 51 mild, 14 moderate and 1 severe \\
\hline $\begin{array}{l}\text { Gonzalez- } \\
\quad \text { Moran } \\
{[43]}\end{array}$ & 28 & 31 & 0 & 3 & NR & NR & NR & $\begin{array}{l}4 \text { wound infection } \\
9 \text { metalware } \\
\text { problems }\end{array}$ & $\begin{array}{l}1 \text { acute, } 4 \text { acute on chronic and } 26 \text { chronic } \\
0 \text { preslip, } 15 \text { mild, } 12 \text { moderate and } 4 \text { severe }\end{array}$ \\
\hline $\begin{array}{l}\text { Zahrawi } \\
\text { [42] }\end{array}$ & NR & 60 & 0 & 2 & NR & NR & $\begin{array}{l}49 \text { excellent } \\
6 \text { good } \\
0 \text { fair } \\
3 \text { poor } \\
2 \text { failure }\end{array}$ & $\begin{array}{l}2 \text { metalware } \\
\text { problems } \\
1 \text { further slipping } \\
3 \text { wound infection } \\
2 \text { needed further } \\
\text { surgery }\end{array}$ & $\begin{array}{l}\text { Severity (mean slip angle 22) } \\
\text { Chronicity and stability NR } \\
\text { LOS } 17 \\
\text { Duration of surgery } 90 \mathrm{~min} \\
\text { Blood loss } 250 \mathrm{ml} \\
\text { HHC }\end{array}$ \\
\hline Total & NR & 273 & 6 & 11 & & & $\begin{array}{l}76(67 \%) \\
\text { excellent } \\
19(17 \%) \\
\text { good } \\
0(0 \%) \text { fair } \\
16(14 \%) \\
\text { poor } \\
3(3 \%) \\
\text { failure }\end{array}$ & & $\begin{array}{l}\text { AVN rate } 2.2 \%(6 / 273) \\
\text { CL rate } 4 \%(11 / 273) \\
\text { FAI }(\mathrm{NR}) \\
\text { OA } 15 \%(18 / 120)\end{array}$ \\
\hline
\end{tabular}

$A V N$ femoral head osteonecrosis, $C L$ chondrolysis, $C R I F$ closed reduction and internal fixation, $N R$ not reported or suboptimum reporting to provide useful information, HHC Heyman and Herndon classification, IHS Iowa hip-rating system; excellent 90-100 points; good 80-89 points; fair 70-79 points; and poor 470points, LOS length of stay, FAI femora-acetabular impingement, $O A$ osteoarthritis

a Satisfactory patients result based on closely related ratings such as Heyman and Herndon classification, Harris hip score or Iowa hip scores

shows a pooled summary of the AVN rates among various interventions.

\section{Femoro-acetabular impingement}

Six studies (324 hips) provided useful data on the rate of FAI [43, 46, 57, 58, 70, 71]. These studies investigated pinning in situ, physeal osteotomy and Ganz surgical dislocation. The FAI rates were $29.8,1.5$ and $6 \%$, respectively. The difference was statistically significant [Fisher exact test $(d f=2): P<0.001$ ]. One study [71] reported the presence of radiological signs of FAI in 30 of 37 hips that were treated with pinning in situ. The study was not explicit about their impingement symptoms. Sensitivity analysis by excluding the study showed that the FAI rate was 7, 1.5 and $6 \%$, respectively; a nonsignificant difference [Fisher exact test $(d f=2)$ : $P=0.13]$. 
Table 8 Studies of physeal osteotomy in stable slip

\begin{tabular}{|c|c|c|c|c|c|c|c|c|c|}
\hline Study & Patients & Hips & AVN & CL & FAI & $\mathrm{OA}$ & $\begin{array}{l}\text { Patient } \\
\text { satisfaction }^{\mathrm{a}}\end{array}$ & $\begin{array}{l}\text { Other } \\
\text { complications }\end{array}$ & Notes \\
\hline $\begin{array}{l}\text { Abu } \\
\text { Amara } \\
{[65]}\end{array}$ & NR & 44 & 4 & 1 & 0 & NR & & & $\begin{array}{l}\text { See also PIS } \\
92 \text { unstable slips were excluded } \\
\text { WOMAC (3.8) } \\
\text { HHS }(92.5)\end{array}$ \\
\hline $\begin{array}{l}\text { Alshryda } \\
\text { [3] }\end{array}$ & 7 & 7 & 2 & 1 & NR & NR & NR & Hip dislocation & 15 unstable hips were excluded ( 5 AVN) \\
\hline Barros [33] & 23 & 23 & 3 & 2 & NR & NR & $\begin{array}{l}9 \text { excellent } \\
9 \text { good } \\
1 \text { fair } \\
4 \text { poor }\end{array}$ & $\begin{array}{l}1 \text { metalware } \\
\text { problem } \\
0 \text { infection }\end{array}$ & $\begin{array}{l}0 \text { acute, } 3 \text { acute on chronic, } 20 \text { chronic } \\
0 \text { mild, } 0 \text { moderate, } 23 \text { severe } \\
\text { MSC }\end{array}$ \\
\hline $\begin{array}{l}\text { Broughton } \\
\text { [57] }\end{array}$ & 115 & 115 & 14 & 14 & 1 & 17 & $\begin{array}{l}67 \text { good } \\
9 \text { fair } \\
19 \text { poor }\end{array}$ & & $\begin{array}{l}0 \text { acute, } 38 \text { acute on chronic, } 77 \text { chronic } \\
0 \text { mild, } 15 \text { moderate, } 100 \text { severe } \\
\text { Patients satisfaction }(\mathrm{G} / \mathrm{F} / \mathrm{B}) \text { in the acute on chronic } \\
(27 / 5 / 6) \text {; in the chronic with open growth plate } \\
(59 / 3 / 8) \text { in the chronic slip with closed growth } \\
\text { plate }(1 / 1 / 5)\end{array}$ \\
\hline $\begin{array}{c}\text { Carlioz } \\
{[30]}\end{array}$ & 26 & 27 & 0 & 3 & NR & NR & $\begin{array}{l}20 \text { good } \\
3 \text { fair } \\
4 \text { bad } \\
3 \text { failure }\end{array}$ & Septic arthritis & \\
\hline $\begin{array}{c}\text { Carney } \\
{[35]}\end{array}$ & NR & 14 & 3 & 6 & NR & NR & NR & & $\begin{array}{l}26 \text { moderate or severe slips } \\
\text { IHS for chronic slips } 76 \text { and } 50 \text { for acute slips }\end{array}$ \\
\hline $\begin{array}{l}\text { DeRosa } \\
{[70]}\end{array}$ & 23 & 27 & 4 & 8 & NR & NR & $\begin{array}{l}0 \text { excellent } \\
19 \text { good } \\
4 \text { fair } \\
4 \text { poor }\end{array}$ & 2 loss of fixation & $\begin{array}{l}1 \text { CRIF before PO went into AVN } \\
0 \text { mild, } 0 \text { moderate, } 27 \text { severe } \\
\text { MSC }\end{array}$ \\
\hline $\begin{array}{l}\text { Dreghorn } \\
{[56]}\end{array}$ & NR & 3 & 1 & 0 & NR & 0 & NR & $\begin{array}{l}1 \text { wound } \\
\text { infection }\end{array}$ & 0 mild, 5 moderate and 6 severe \\
\hline Diab [58] & 11 & 11 & 2 & 0 & 1 & NR & NR & & \\
\hline Dunn [59] & 69 & 73 & 9 & 3 & NR & 2 & $\begin{array}{l}55 \text { good } \\
6 \text { fair } \\
12 \text { poor }\end{array}$ & & $\begin{array}{l}\text { Several hips were manipulated under GA } \\
\text { somewhere else (CRIF) } \\
0 \text { acute, } 33 \text { acute on chronic, } 40 \text { chronic }\end{array}$ \\
\hline Fish [60] & 61 & 66 & 3 & 1 & NR & 6 & $\begin{array}{l}55 \text { excellent } \\
6 \text { good } \\
2 \text { fair } \\
3 \text { poor }\end{array}$ & & $\begin{array}{l}0 \text { acute, } 16 \text { acute on chronic, } 50 \text { chronic } \\
\text { Chronic slips ( } 0 \text { mild, } 23 \text { moderate, } 27 \text { severe) }\end{array}$ \\
\hline Fron $[62]$ & 46 & 50 & 6 & 3 & NR & NR & $\begin{array}{l}34 \text { excellent } \\
10 \text { good } \\
2 \text { fair } \\
4 \text { poor }\end{array}$ & $\begin{array}{l}2 \text { hematomas } \\
2 \text { infections } \\
3 \text { pseudarthroses } \\
\text { of the greater } \\
\text { trochanter } 1 \\
\text { HO }\end{array}$ & $\begin{array}{l}0 \text { acute, } 17 \text { acute on chronic, } 30 \text { chronic } \\
0 \text { mild, } 0 \text { moderate, } 50 \text { severe }\end{array}$ \\
\hline Jerre [63] & 22 & 22 & 5 & 1 & NR & 6 & $\begin{array}{l}5 \text { excellent } \\
4 \text { good } \\
1 \text { fair } \\
8 \text { poor }\end{array}$ & $\begin{array}{l}4 \text { THR } \\
1 \text { hip arthrodesis }\end{array}$ & $\begin{array}{l}1 \text { acute, } 1 \text { acute on chronic, } 20 \text { chronic } \\
10 \text { mild, } 6 \text { moderate, } 0 \text { severe, } 6 \text { none } \\
\text { HHC }\end{array}$ \\
\hline
\end{tabular}


Table 8 continued

\begin{tabular}{|c|c|c|c|c|c|c|c|c|c|}
\hline Study & Patients & Hips & AVN & CL & FAI & $\mathrm{OA}$ & $\begin{array}{l}\text { Patient } \\
\text { satisfaction }^{\mathrm{a}}\end{array}$ & $\begin{array}{l}\text { Other } \\
\text { complications }\end{array}$ & Notes \\
\hline Niane [64] & 24 & 26 & 1 & 5 & NR & NR & $\begin{array}{l}20 \text { excellent } \\
\text { and good } \\
6 \text { poor }\end{array}$ & $\begin{array}{l}\text { The Postel Merle } \\
\text { d'Aubigné } \\
\text { (PMA) }\end{array}$ & $\begin{array}{l}\text { Severity grade II and III only (grade I were } \\
\text { excluded) }\end{array}$ \\
\hline $\begin{array}{l}\text { Nishiyama } \\
{[65]}\end{array}$ & 15 & 18 & 1 & 1 & & & $\begin{array}{l}13 \text { excellent } \\
1 \text { good } \\
1 \text { fair } \\
0 \text { poor }\end{array}$ & & $\begin{array}{l}0 \text { acute, } 0 \text { acute on chronic, } 18 \text { chronic } \\
0 \text { mild, } 0 \text { moderate, } 18 \text { severe }\end{array}$ \\
\hline $\begin{array}{l}\text { Szypryt } \\
{[41]}\end{array}$ & 23 & 23 & 4 & 0 & NR & 5 & $\begin{array}{l}15 \text { excellent } \\
2 \text { good } \\
1 \text { fair } \\
4 \text { poor }\end{array}$ & $\begin{array}{l}2 \text { wound } \\
\text { infection } \\
\text { Metalware } \\
\text { problems } 10\end{array}$ & $\begin{array}{l}1 \text { acute, } 16 \text { acute on chronic, } 6 \text { chronic } \\
0 \text { mild, } 0 \text { moderate, } 23 \text { severe } \\
\text { MSC }\end{array}$ \\
\hline $\begin{array}{l}\text { Velasco } \\
{[66]}\end{array}$ & 65 & 66 & 6 & 8 & & & $\begin{array}{l}22 \text { good } \\
16 \\
\text { moderate } \\
\text { (fair) } \\
10 \text { poor }\end{array}$ & & $\begin{array}{l}8 \text { acute, } 29 \text { acute on chronic, } 29 \text { chronic } \\
\text { All moderate or severe (although Table } 2 \text { showed } \\
\text { that angles }<30^{\circ} \text { in five hips) } \\
\text { Full set data in } 48 \text { hips }\end{array}$ \\
\hline Total & NR & 615 & 68 & 57 & & & $\begin{array}{l}141 \\
\text { excellent } \\
220 \text { good } \\
46 \text { fair } \\
78 \text { poor } \\
3(6 \%) \\
\text { failure }\end{array}$ & & $\begin{array}{l}\text { AVN rate } 10.4 \%(64 / 615) \\
\text { CL rate } 9.2 \%(57 / 615) \\
\text { FAI rate } 1.2 \%(2 / 170) \\
\text { OA rate } 12.2(36 / 294)\end{array}$ \\
\hline
\end{tabular}

$A V N$ femoral head osteonecrosis, $C L$ chondrolysis, $C R I F$ closed reduction and internal fixation, $N R$ not reported or suboptimum reporting to provide useful information, HHS Harris hip score or modified Harris hip score; excellent 90-100 points; good 80-89 points; fair 70-79 points; and poor $<70$ points. HHC Heyman and Herndon classification, IHS Iowa hip-rating system; excellent 90-100 points; good 80-89 points; fair 70-79 points; and poor $<70$ points. MSC modified southwick criteria, WOMAC Western Ontario and McMaster Universities Osteoarthritis Index, FAI femora-acetabular impingement, $O A$ osteoarthritis, THR total hip replacement

a Satisfactory patients result based on closely related ratings such as Heyman and Herndon classification, Harris hip score or Iowa hip scores

\section{Osteoarthritis}

The overall OA rate was $11 \%$ with the lowest rate seen in patients who underwent Ganz surgical dislocation (0\%), followed by PIS (3.1\%). Hip spica was associated with the highest $\mathrm{OA}$ rate $(52 \%)$. The variation in the OA rates among various interventions difference was statistically significant [Fisher exact test $(d f=5)$ : $P<0.001]$.

\section{Patient satisfaction rates}

Patient satisfaction scores were reported for all interventions apart from hip spica. Most included studies used closely related scores which were categorised into an ordinal scale of (excellent, good, fair, poor and failure). These are summarised in Fig. 2. Visual analysis of the graphs favours Ganz surgical dislocation.

\section{Continued growth}

Three studies reported on screws that allow for continued growth. Guzzanti [23] reported on 12 patients (6 with stable SCFE) who were treated using a modified AO cannulated screw (HIT-MEDICA, Rimini, Italy). The distal segment has 3 rather than the original six threads. Holmdahl [55] reported on 13 patients (10 with stable SCFE) who were treated with Hansson hook pins. The Hansson hook pin is a smooth $6.5-\mathrm{mm}$-diameter pin with a central hook that offers secure anchorage in the epiphysis and the smooth pin allows the femoral neck to continue to grow. Wensaas [54] reported on 14 patients (16 stable SCFE) who were treated with a modified Olmeda screw (De Puy). The screw has a shorter thread segment. The three studies used various measures to assess growth and remodelling. They showed that growth and remodelling continued when these screws were used. 
Table 9 Studies of Ganz surgical dislocation in stable slips

\begin{tabular}{|c|c|c|c|c|c|c|c|c|c|}
\hline Study & Patients & Hips & AVN & $\mathrm{CL}$ & FAI & OA & $\begin{array}{l}\text { Patient } \\
\text { satisfaction }^{\mathrm{a}}\end{array}$ & Other complications & Notes \\
\hline Bali [67] & 8 & 8 & 0 & 0 & NR & NR & NR & $\begin{array}{l}2 \text { non-unions requiring valgus } \\
\text { intertrochanteric } \\
\text { osteotomies }\end{array}$ & HHS: 92.5 \\
\hline $\begin{array}{l}\text { Dan } \\
\text { Cosma } \\
{[43]}\end{array}$ & 6 & 6 & 0 & 0 & 1 & NR & $\begin{array}{r}6 \text { excellent } \\
\text { and good }\end{array}$ & & $\begin{array}{l}\text { One unstable slip excluded } \\
10 \text { pinned in situ } \\
\text { HHC }\end{array}$ \\
\hline $\begin{array}{c}\text { Madan } \\
{[68]}\end{array}$ & 11 & 11 & 0 & 1 & NR & NR & NR & & $\begin{array}{l}17 \text { unstable hips were excluded ( } 4 \text { AVN) } \\
0 \text { acute, } 0 \text { acute-on-chronic, } 11 \text { chronic } \\
3 \text { had previous operations } \\
\text { HHS }(90.3) \\
\text { NAHS }(91.0)\end{array}$ \\
\hline $\begin{array}{r}\text { Masse } \\
{[69]}\end{array}$ & 18 & 18 & 0 & 0 & & 0 & $\begin{array}{l}18 \text { excellent } \\
0 \text { good } \\
0 \text { fair } \\
0 \text { poor } \\
0 \text { failure }\end{array}$ & 1 metalware problem & $\begin{array}{l}2 \text { unstable hips excluded (no AVN) } \\
2 \text { mild, } 4 \text { moderate, } 12 \text { severe } \\
\text { HHS (98.2) }\end{array}$ \\
\hline $\begin{array}{c}\text { Novais } \\
{[51]}\end{array}$ & 15 & 15 & 1 & NR & NR & NR & $\begin{array}{l}7 \text { excellent } \\
2 \text { good } \\
0 \text { fair } \\
5 \text { poor } \\
1 \text { failure }\end{array}$ & 2 metalware problems & $\mathrm{HHC}$ \\
\hline $\begin{array}{c}\text { Souder } \\
{[52]}\end{array}$ & NR & 10 & 2 & 1 & NR & NR & NR & 1 metalware problem & $\begin{array}{l}\text { From a total of } 17 \text { hips, } 7 \text { were unstable, } \\
2 \text { of these unstable hips went into AVN }\end{array}$ \\
\hline $\begin{array}{l}\text { Ziebarth } \\
\text { [70] }\end{array}$ & 27 & 27 & 0 & 0 & 1 & & $\begin{array}{l}27 \text { excellent } \\
0 \text { good } \\
0 \text { fair } \\
0 \text { poor } \\
0 \text { failure }\end{array}$ & & $\begin{array}{l}25 \text { patients from series A and } 2 \text { from } \\
\text { series B } \\
5 \text { unstable/uncertain hips excluded } \\
0 \text { mild, } 15 \text { moderate, } 12 \text { severe } \\
\text { HHS }(96.5)\end{array}$ \\
\hline Total & NR & 95 & 3 & 2 & & & $\begin{array}{l}52(87 \%) \\
\text { excellent } \\
2(3 \%) \\
\text { good } \\
0(0 \%) \text { fair } \\
5(8 \%) \text { poor } \\
1(2 \%) \\
\text { failure }\end{array}$ & & $\begin{array}{l}\text { AVN rate } 3.1 \%(3 / 95) \\
\text { CL rate } 2.5 \%(2 / 80) \\
\text { FAI rate } 6.1 \%(2 / 33) \\
\text { OA rate } 0 \%(0 / 18)\end{array}$ \\
\hline
\end{tabular}

$A V N$ femoral head osteonecrosis, $C L$ chondrolysis, $N R$ not reported or suboptimum reporting to provide useful information, $H H S$ Harris hip score or modified Harris hip score; excellent 90-100 points; good 80-89 points; fair 70-79points; and poor < 70 points. HHC Heyman and Herndon classification, NAHS non arthritic hip scores, FAI femora-acetabular impingement, $O A$ osteoarthritis

a Satisfactory patients result based on closely related ratings such as Heyman and Herndon classification, Harris hip score or Iowa hip scores

\section{Discussion}

Several aspects of slipped capital femoral epiphysis remain controversial. Loder's work has been instrumental in changing our understanding and approach to the management of the condition when he introduced the concept of "slip instability", which was fundamental in better understanding certain aspects of the condition. Two different types of SCFE became apparent; unstable slips where the patient cannot ambulate even with crutches, and stable slips where the patient can ambulate. Loder showed that AVN developed in $47 \%$ of unstable slips but none of 


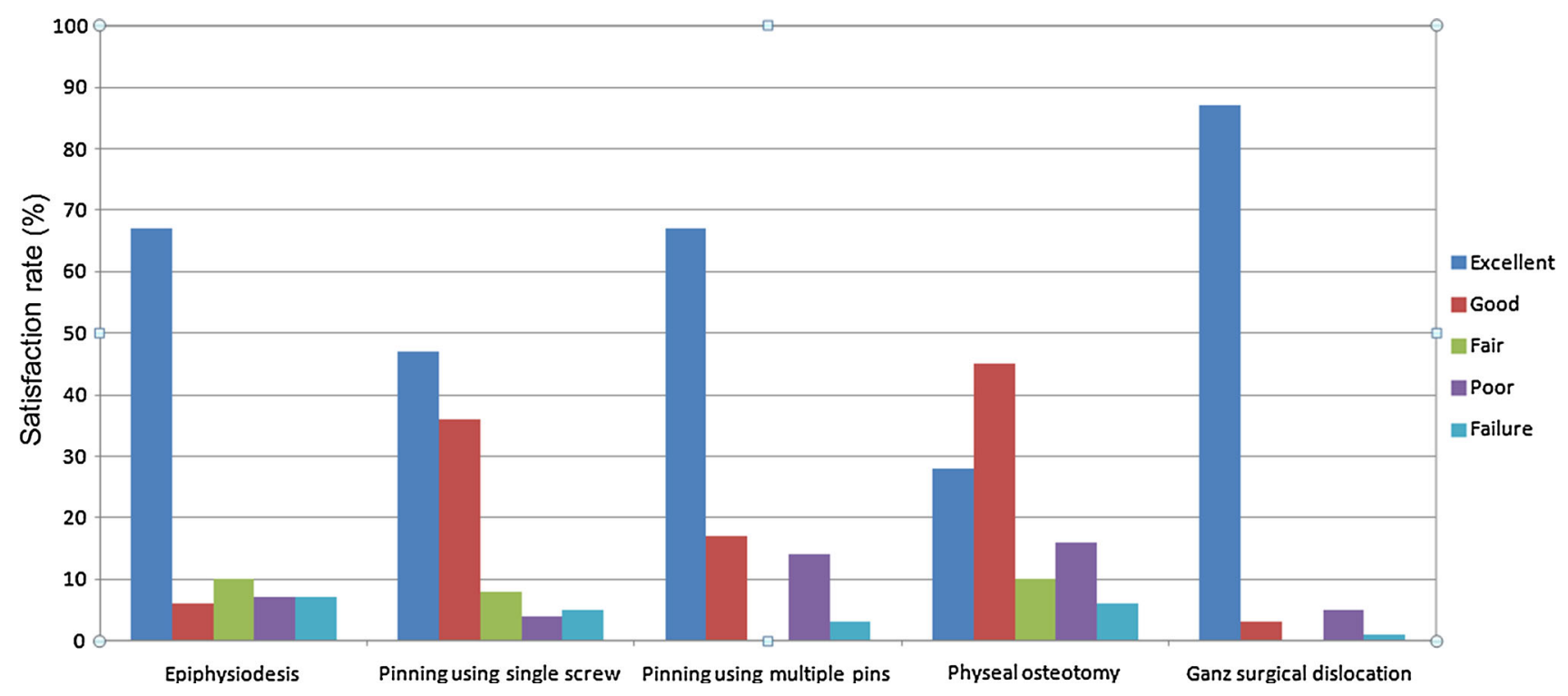

Fig. 2 Satisfaction rates among various interventions to treat slipped capital femoral epiphysis

the stable hips. This finding has been replicated by others [3-6, 71].

These two types of SCFE behave differently in terms of presentation, progress and complications; hence treatments are likely to be different. For this reason, we approached them as two different conditions to highlight these differences [26]. In our previously published review, open reduction and internal fixation using the Parsch technique $[26,72]$ stood out as the best current technique to treat unstable slipped capital femoral epiphysis. In this review, five outcomes were analysed to compare various interventions: AVN, CL, FAI, OA and patient satisfaction rates. Pinning in situ and Ganz surgical dislocation are shown to be superior to other interventions in treating stable slips (Table 3).

The review confirmed that the rate of AVN in stable slips is much lower than that in unstable slips (5.1 versus $21 \%$ ) [26]. The type of surgical intervention is an important risk factor. Pinning in situ was associated with the lowest AVN rate (1.4\%). Moreover, the CL, FAI and OA rates were relatively low in patients who underwent PIS. These were not translated into high patient satisfaction rates among these patients, with only $47 \%$ reporting an "excellent" outcome. In contrast, $87 \%$ of patients who underwent Ganz surgical dislocation reported an "excellent" outcome. The Ganz surgical dislocation was associated with an AVN rate of 3.3\%; double that observed in pinning in situ. Of note, 5 of the 7 studies which investigated the Ganz dislocation reported a AVN rate of $0 \%$. The overall mean AVN rate for this technique is hence derived from the two remaining studies and therefore the actual AVN rate for this procedure may in reality be lower than $3.3 \%$. As a relatively new procedure and with small patient numbers, there may also be a substantial learning curve associated with this technique.

When non-threaded pins and wires were used, the neck commonly continues to grow and this would be a great advantage. However, stabilisation using multiple pins was not found to provide advantages over pinning using a single screw, with substantially higher AVN, CL, FAI and OA rates. Moreover, with continued growth there is a risk that the anchorage in the epiphysis will be lost and repeat fixation will be required. Further growth of the femoral neck is less likely to occur if a screw is inserted in compression mode with the head abutting the lateral femoral cortex, causing physiodesis [73]. Three studies $[23,54,55]$ showed that screws with special design allowed growth to continue; however, these were small studies (37 patients) with no comparator. The literature search identified 6 other studies that used screws which allow continued growth and reported a favourable outcome on neck growth; however, these studies could not be included in our review because we were not certain about the stability of the slips.

Physeal osteotomy using Fish or Dunn techniques was associated with high AVN, CL, FAI and OA rates and only $28 \%$ reported an excellent outcome. Bone peg epiphysiodesis has not been favoured by the orthopaedic community because of the associated blood loss, donor site morbidity, length of surgical time and length of stay. Although the AVN and CL rates were relatively low, patients' satisfaction rates were not impressive with only $67 \%$ reporting an excellent outcome and $14 \%$ reporting a poor outcome or failure. Although the reported OA rate was high (23.3\%), this was derived from a single study which could be an outlier. 
Hip spica was found to be the worst treatment, with poor AVN (9\%), CL (20.5\%) and OA (53\%) rates. This probably explains why this treatment modality has largely been abandoned in the management of SCFE.

Timing of surgery and severity of the slip are two factors that we intended to study; however, included studies did not provide useful data to inform the effect of these factors on the selected outcomes. It is our observation that timing of surgery is not as critical as in unstable slips but the severity of slips plays a role in final outcomes and patient satisfaction rates. However, this remains to be proven.

The review was conducted with the intention of doing a trial-based meta-analysis. Disappointingly, only case series and controlled studies were found and form the basis of this review. Some studies were published more than once with or without extra information. Thorough considerations have been taken when including data from such studies. Authors were often contacted for further clarification and data provision. Despite our best effort to produce a high quality review, the qualities of the included studies remain the major weakness of this review. Yet, this is the largest systematic review that has addressed this hot topic and which explains the current trends in treating slipped capital femoral epiphysis.

Another inherent bias that must be considered when reading our findings is the fact that pinning in situ was used across the whole severity spectrum of SCFE and this was not the case with Ganz surgical dislocation. Ganz surgical dislocation is more invasive than PIS and has been reserved for patients with severe stable slips. In such cases, the merits and risks of this technique should be discussed with the parents: an excellent reported patient satisfaction but a higher incidence of AVN, respectively.

In summary, the review supported our views that stable and unstable slips behave differently and require different treatments. For an unstable SCFE, open reduction and internal fixation on an urgent basis (within $24 \mathrm{~h}$ ) is shown to be associated with the best outcome [26]. For a stable SCFE, pinning in situ is recommended for mild and, to a lesser extent, moderate slips. Screws that allow continued growth may be superior to standard screws. Ganz surgical dislocation is recommended for severe slips provided patients and parents agree to take the higher AVN risk for better satisfaction and the surgical expertise is available.

\section{Compliance with ethical standards}

Conflict of interest All authors declare no conflict of interest.

Patient consent This study did not involve human or animal research, hence no patient consent was required.

Ethical approval This study was conducted in accordance with the ethical standards of the institutional and/or national research committee and with the 1964 Helsinki Declaration and its later amendments or comparable ethical standards.

Funding For this study, there was no source of funding.

Open Access This article is distributed under the terms of the Creative Commons Attribution 4.0 International License (http://crea tivecommons.org/licenses/by/4.0/), which permits unrestricted use, distribution, and reproduction in any medium, provided you give appropriate credit to the original author(s) and the source, provide a link to the Creative Commons license, and indicate if changes were made.

\section{References}

1. Alshryda S, Jones S, Banaszkiewicz P (2014) Postgraduate paediatric orthopaedics: the candidate's guide to the FRCS (Tr and Orth) examination. Cambridge University Press, Cambridge

2. Loder RT, Richards BS, Shapiro PS, Reznick LR, Aronson DD (1993) Acute slipped capital femoral epiphysis: the importance of physeal stability. J Bone Jt Surg Am 75(8):1134-1140

3. Alshryda S, Tsang K, Ahmed M, Adedapo A, Montgomery R (2013) Severe slipped upper femoral epiphysis; fish osteotomy versus pinning in situ: an eleven-year perspective. Surgeon 12(5):244-248

4. Alves C, Steele M, Narayanan U, Howard A, Alman B, Wright JG (2013) Open reduction and internal fixation of unstable slipped capital femoral epiphysis by means of surgical dislocation does not decrease the rate of avascular necrosis: a preliminary study. J Child Orthop 6(4):277-283

5. Aronsson DD, Loder RT (1996) Treatment of the unstable (acute) slipped capital femoral epiphysis. Clin Orthop Relat Res 322:99-110

6. Kalogrianitis S, Tan CK, Kemp GJ, Bass A, Bruce C (2007) Does unstable slipped capital femoral epiphysis require urgent stabilization? J Pediatr Orthop B 16(1):6-9

7. Loder RT (2008) Correlation of radiographic changes with disease severity and demographic variables in children with stable slipped capital femoral epiphysis. J Pediatr Orthop 28(3):284-290

8. Loder RT (2006) Controversies in slipped capital femoral epiphysis. Orthop Clin N Am 37(2):211-21, vii

9. Loder RT (2001) Unstable slipped capital femoral epiphysis. J Pediatr Orthop 21(5):694-699

10. Loder RT (2001) Effect of femur position on the angular measurement of slipped capital femoral epiphysis. J Pediatr Orthop 21(4):488-494

11. Loder RT (1996) The demographics of slipped capital femoral epiphysis. An international multicenter study. Clin Orthop Relat Res 322:8-27

12. Loder RT (1996) A worldwide study on the seasonal variation of slipped capital femoral epiphysis. Clin Orthop Relat Res 322:28-36

13. Loder RT (1995) Slipped capital femoral epiphysis in children. Curr Opin Pediatr 7(1):95-97

14. Loder RT, Aronson DD, Greenfield ML (1993) The epidemiology of bilateral slipped capital femoral epiphysis. A study of children in Michigan. J Bone Jt Surg Am 75(8):1141-1147

15. Loder RT, Farley FA, Herzenberg JE, Hensinger RN, Kuhn JL (1993) Narrow window of bone age in children with slipped capital femoral epiphyses. J Pediatr Orthop 13(3):290-293

16. Loder RT, Greenfield ML (2001) Clinical characteristics of children with atypical and idiopathic slipped capital femoral epiphysis: description of the age-weight test and implications for further diagnostic investigation. J Pediatr Orthop 21(4):481-487 
17. Loder RT, Hensinger RN (1997) Slipped capital femoral epiphysis associated with renal failure osteodystrophy. J Pediatr Orthop 17(2):205-211

18. Loder RT, Hensinger RN, Alburger PD, Aronsson DD, Beaty JH, Roy DR et al (1998) Slipped capital femoral epiphysis associated with radiation therapy. J Pediatr Orthop 18(5): 630-636

19. Loder RT, Mehbod AA, Meyer C, Meisterling M (2003) Acetabular depth and race in young adults: a potential explanation of the differences in the prevalence of slipped capital femoral epiphysis between different racial groups? J Pediatr Orthop 23(6):699-702

20. Loder RT, Starnes T, Dikos G (2006) Atypical and typical (idiopathic) slipped capital femoral epiphysis. Reconfirmation of the age-weight test and description of the height and age-height tests. J Bone Jt Surg Am 88(7):1574-1581

21. Hansson LI (1982) Osteosynthesis with the hook-pin in slipped capital femoral epiphysis. Acta Orthop Scand 53(1):87-96

22. Falciglia F, Aulisa AG, Giordano M, Boldrini R, Guzzanti V (2010) Slipped capital femoral epiphysis: an ultrastructural study before and after osteosynthesis. Acta Orthop 81(3):331-336

23. Guzzanti V, Falciglia F, Stanitski CL (2004) Slipped capital femoral epiphysis in skeletally immature patients. J Bone Jt Surg Br 86(5):731-736

24. Guzzanti V, Falciglia F, Stanitski CL, Stanitski DF (2003) Slipped capital femoral epiphysis: physeal histologic features before and after fixation. J Pediatr Orthop 23(5):571-577

25. Perry D (2016) The British Orthopaedic Surgery Surveillance 2016. http://boss.surgery/. Accessed July 2017

26. Alshryda S, Tsang K, Chytas A, Chaudhry M, Sacchi K, Ahmad M, Mason JM (2016) Evidence based treatment for unstable slipped upper femoral epiphysis: systematic review and exploratory patient level analysis. Surgeon. doi:10.1016/j.surge.2016.04.001

27. Alshryda S, Tsang K, Al-Shryda J, Blenkinsopp J, Adedapo A, Montgomery R et al (2013) Interventions for treating slipped upper femoral epiphysis (SUFE). Cochrane Database Syst Rev. doi:10.1002/14651858CD010397

28. Moher D, Liberati A, Tetzlaff J, Altman DG (2009) Preferred reporting items for systematic reviews and meta-analyses: the PRISMA statement. BMJ 339:b2535

29. Alshryda S, Wright J (2014) Acute slipped capital femoral epiphysis: the importance of physeal stability. Classic papers in orthopaedics, 2014. Springer, London, pp 547-548

30. Carlioz H, Vogt JC, Barba L, Doursounian L (1984) Treatment of slipped upper femoral epiphysis: 80 cases operated on over 10 years (1968-1978). J Pediatr Orthop 4(2):153-161

31. Escott BG, De La Rocha A, Jo CH, Sucato DJ, Karol LA (2015) Patient-reported health outcomes after in situ percutaneous fixation for slipped capital femoral epiphysis: an average twenty-year follow-up study. J Bone Jt Surg Am 97(23):1929-1934

32. Kenny P, Higgins T, Sedhom M, Dowling F, Moore DP, Fogarty EE (2003) Slipped upper femoral epiphysis. A retrospective, clinical and radiological study of fixation with a single screw. J Pediatr Orthop B 12(2):97-99

33. Barros JW, Tukiama G, Fontoura C, Barsam NH, Pereira ES (2000) Trapezoid osteotomy for slipped capital femoral epiphysis. Int Orthop 24(2):83-87

34. Betz RR, Steel HH, Emper WD, Huss GK, Clancy M (1990) Treatment of slipped capital femoral epiphysis. Spica-cast immobilization. J Bone Jt Surg Am 72(4):587-600

35. Carney BT, Weinstein SL, Noble J (1991) Long-term follow-up of slipped capital femoral epiphysis. J Bone Jt Surg Am 73(5):667-674

36. Meier MC, Meyer LC, Ferguson RL (1992) Treatment of slipped capital femoral epiphysis with a spica cast. J Bone Jt Surg Am 74(10):1522-1529
37. Rao SB, Crawford AH, Burger RR, Roy DR (1996) Open bone peg epiphysiodesis for slipped capital femoral epiphysis. J Pediatr Orthop 16(1):37-48

38. Adamczyk MJ, Weiner DS, Hawk D (2003) A 50-year experience with bone graft epiphysiodesis in the treatment of slipped capital femoral epiphysis. J Pediatr Orthop 23(5):578-583

39. Murray T, Morscher MA, Krahe AM, Adamczyk MJ, Weiner DS (2016) Fibular allograft and demineralized bone matrix for the treatment of slipped capital femoral epiphysis. Orthopedics 39(3):e519-e525

40. Schmidt TL, Cimino WG, Seidel FG (1996) Allograft epiphysiodesis for slipped capital femoral epiphysis. Clin Orthop Relat Res 322:61-76

41. Szypryt EP, Clement DA, Colton CL (1987) Open reduction or epiphysiodesis for slipped upper femoral epiphysis. A comparison of Dunn's operation and the Heyman-Herndon procedure. J Bone Jt Surg Br 69(5):737-742

42. Zahrawi FB, Stephens TL, Spencer GE Jr, Clough JM (1983) Comparative study of pinning in situ and open epiphysiodesis in 105 patients with slipped capital femoral epiphyses. Clin Orthop Relat Res 177:160-168

43. Cosma D, Vasilescu DE, Corbu A, Valeanu M, Vasilescu D (2016) The modified Dunn procedure for slipped capital femoral epiphysis does not reduce the length of the femoral neck. Pak J Med Sci 32(2):379-384

44. Aronson DD, Loder RT (1992) Slipped capital femoral epiphysis in black children. J Pediatr Orthop 12(1):74-79

45. Blanco JS, Taylor B, Johnston CE 2nd (1992) Comparison of single pin versus multiple pin fixation in treatment of slipped capital femoral epiphysis. J Pediatr Orthop 12(3):384-389

46. de Poorter JJ, Beunder TJ, Gareb B, Oostenbroek HJ, Bessems GH, van der Lugt JC, Maathuis PG, van der Sande MA (2016) Long-term outcomes of slipped capital femoral epiphysis treated with in situ pinning. J Child Orthop 10(5):371-379. doi:10.1007/ s11832-016-0759-z

47. Gonzalez-Moran G, Carsi B, Abril JC, Albinana J (1998) Results after preoperative traction and pinning in slipped capital femoral epiphysis: K wires versus cannulated screws. J Pediatr Orthop B 7(1):53-58

48. Herman MJ, Dormans JP, Davidson RS, Drummond DS, Gregg JR (1996) Screw fixation of grade III slipped capital femoral epiphysis. Clin Orthop Relat Res (322):77-85

49. Koval KJ, Lehman WB, Rose D, Koval RP, Granft A, Strongwater A (1989) Treatment of slipped capital femoral epiphysis with a cannulated-screw technique. J Bone Jt Surg Am 71(9):1370-1377

50. Lim YJ, Lam KS, Lim KB, Mahadev A, Lee EH (2007) Management outcome and the role of manipulation in slipped capital femoral epiphysis. J Orthop Surg (Hong Kong) 15(3):334-338

51. Novais EN, Hill MK, Carry PM, Heare TC, Sink EL (2014) Modified Dunn procedure is superior to in situ pinning for shortterm clinical and radiographic improvement in severe stable SCFE. Clin Orthop Relat Res 473(6):2108-2117

52. Souder CD, Bomar JD, Wenger DR (2014) The role of capital realignment versus in situ stabilization for the treatment of slipped capital femoral epiphysis. J Pediatr Orthop 34(8):791-798

53. Ward WT, Stefko J, Wood KB, Stanitski CL (1992) Fixation with a single screw for slipped capital femoral epiphysis. J Bone Jt Surg Am 74(6):799-809

54. Wensaas A, Svenningsen S (2005) Slipped capital femoral epiphysis treated with a specially designed screw. Tidsskr Nor Laegeforen 125(20):2788-2790

55. Holmdahl P, Backteman T, Danielsson A, Karrholm J, Riad J (2016) Continued growth after fixation of slipped capital femoral epiphysis. J Child Orthop 10(6):643-650 
56. Dreghorn CR, Knight D, Mainds CC, Blockey NJ (1987) Slipped upper femoral epiphysis - a review of 12 years of experience in Glasgow (1972-1983). J Pediatr Orthop 7(3):283-287

57. Broughton NS, Todd RC, Dunn DM, Angel JC (1988) Open reduction of the severely slipped upper femoral epiphysis. J Bone Jt Surg Br 70(3):435-439

58. Diab M, Hresko MT, Millis MB (2004) Intertrochanteric versus subcapital osteotomy in slipped capital femoral epiphysis. Clin Orthop Relat Res 427:204-212

59. Dunn DM, Angel JC (1978) Replacement of the femoral head by open operation in severe adolescent slipping of the upper femoral epiphysis. J Bone Jt Surg Br 60-B(3):394-403

60. Fish JB (1994) Cuneiform osteotomy of the femoral neck in the treatment of slipped capital femoral epiphysis. A follow-up note. J Bone Jt Surg Am 76(1):46-59

61. Fish JB (1972) Cuneiform osteotomy in treatment of slipped capital femoral epiphysis. N Y State J Med 72(21):2633-2640

62. Fron D, Forgues D, Mayrargue E, Halimi P, Herbaux B (2000) Follow-up study of severe slipped capital femoral epiphysis treated with Dunn's osteotomy. J Pediatr Orthop 20(3):320-325

63. Jerre R, Hansson GR, Wallin J, Karlsson J (1996) Long-term results after realignment operations for slipped upper femoral epiphysis. J Bone Jt Surg [Br] 78(B):745-750

64. Niane MM, Kinkpe CV, Daffe M, Sarr L, Gueye AB, Sane AD et al (2016) Modified Dunn osteotomy using an anterior approach used to treat 26 cases of SCFE. Orthop Traumatol Surg Res 102(1):81-85

65. Nishiyama K, Sakamaki T, Ishii Y (1989) Follow-up study of slipped capital femoral epiphysis. J Pediatr Orthop 9(6):653-659
66. Velasco R, Schai PA, Exner GU (1998) Slipped capital femoral epiphysis: a long-term follow-up study after open reduction of the femoral head combined with subcapital wedge resection. J Pediatr Orthop B 7(1):43-52

67. Bali K, Railton P, Kiefer GN, Powell JN (2014) Subcapital osteotomy of the femoral neck for patients with healed slipped capital femoral epiphysis. Bone Jt J 96-B(11):1441-1448

68. Madan SS, Cooper AP, Davies AG, Fernandes JA (2013) The treatment of severe slipped capital femoral epiphysis via the Ganz surgical dislocation and anatomical reduction: a prospective study. Bone Jt J 95-B(3):424-429

69. Masse A, Aprato A, Grappiolo G, Turchetto L, Campacci A, Ganz R (2012) Surgical hip dislocation for anatomic reorientation of slipped capital femoral epiphysis: preliminary results. Hip Int 22(2):137-144

70. Ziebarth K, Zilkens C, Spencer S, Leunig M, Ganz R, Kim YJ (2009) Capital realignment for moderate and severe SCFE using a modified Dunn procedure. Clin Orthop Relat Res 467(3):704-716

71. Abu Amara S, Cunin V, Ilharreborde B (2015) Severe slipped capital femoral epiphysis: a French multicenter study of 186 cases performed by the SoFOP. Orthop Traumatol Surg Res 101(6 Suppl):S275-S279

72. Parsch K, Weller S, Parsch D (2009) Open reduction and smooth Kirschner wire fixation for unstable slipped capital femoral epiphysis. J Pediatr Orthop 29(1):1-8

73. Hansson G, Nathorst-Westfelt J (2012) Management of the contralateral hip in patients with unilateral slipped upper femoral epiphysis: to fix or not to fix-consequences of two strategies. J Bone Jt Surg Br 94(5):596-602 